\title{
LPM-Effect in Monte Carlo Models of Radiative Energy Loss
}

\author{
Korinna C. Zapp ${ }^{a, b}$, Johanna Stachel ${ }^{a}$, Urs Achim Wiedemann ${ }^{c}$ \\ ${ }^{a}$ Physikalisches Institut, Universität Heidelberg, Philosophenweg 12, D-69120 Heidelberg, Germany \\ ${ }^{b}$ ExtreMe Matter Institute EMMI, GSI Helmholtzzentrum für Schwerionenforschung GmbH, Planckstraße 1, 64291 \\ Darmstadt, Germany \\ ${ }^{c}$ Physics Department, Theory Unit, CERN, CH-1211 Genève 23, Switzerland
}

\begin{abstract}
Extending the use of Monte Carlo (MC) event generators to jets in nuclear collisions requires a probabilistic implementation of the non-abelian LPM effect. We demonstrate that a local, probabilistic MC implementation based on the concept of formation times can account fully for the LPM-effect. The main features of the analytically known eikonal and collinear approximation can be reproduced, but we show how going beyond this approximation can lead to qualitatively different results.
\end{abstract}

\section{Introduction}

With JEwel (Jet Evolution With Energy Loss) we work towards a dynamically consistent Monte Carlo event generator (MC) for jet quenching that is consistent with all analytically known limiting cases. These limiting cases are the parton shower evolution in the absence of medium effects (as in $e^{+} e^{-}$or $p p$ collisions), energy loss due to elastic scattering in a medium in the absence of vacuum radiation, medium induced gluon emission (radiative energy loss) in the absence of vacuum radiation and to some extent induced gluon emission in the presence of vacuum radiation. The latter is the only part that is not fully implemented in JEWEL yet.

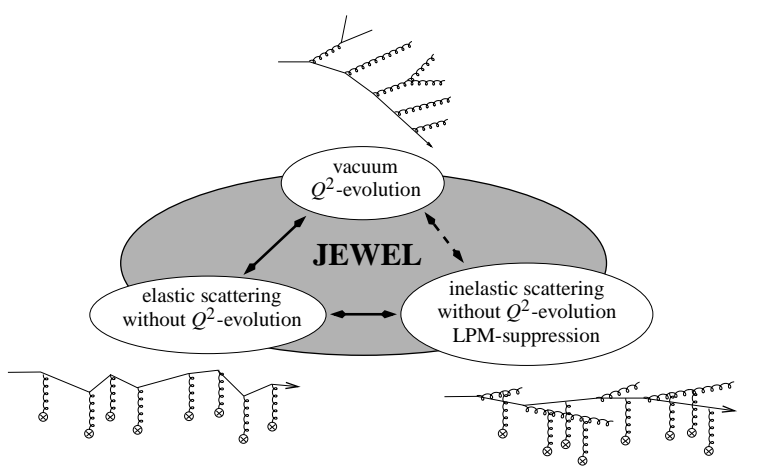

For a detailed discussion of the vacuum parton shower, elastic energy loss and the combination of both we refer to [1]. Here, we focus on the Monte Carlo implementation of medium induced gluon bremsstrahlung, where it is known from analytical calculations that a quantum mechanical interference, namely the non-abelian Landau-Pomerantschuk-Migdal (LPM) effect, plays an important role. 


\section{Reproducing the BDMPS-ASW Results}

Inspection of the field theoretical calculations [2, 3] reveals that radiated gluons have a formation time

$$
t_{\mathrm{f}}=\frac{2 \omega}{k_{\perp}^{2}}
$$

that can be interpreted as the time it takes the gluon to decohere from the radiating projectile, i.e. to build up a relative phase that is of order unity. During this time the gluon may acquire additional transverse momentum due to multiple elastic rescattering, but further gluon emission off the projectile is suppressed. This suggests that the interference can be taken into account by a $\mathrm{MC}$ algorithm that correctly treats the formation time effects [4]. To be more specific, the position of the inelastic process is determined probabilistically and a gluon is produced with energy $\omega$ and transverse momentum $k_{\perp}$. Then the position of the next interaction is chosen. If the distance to this scattering centre is larger than the gluon formation time $t_{\mathrm{f}}$, then the gluon is regarded as fully formed independent particle and the scattering centre can be the source of further gluon radiation. If, on the other hand, the scattering centre is found within the formation time the momentum transfer $q_{\perp}$ is added coherently to the gluon production process. This changes the formation time. If now the next scattering occurs outside the updated formation time the gluon is regarded as formed, otherwise the procedure is repeated.
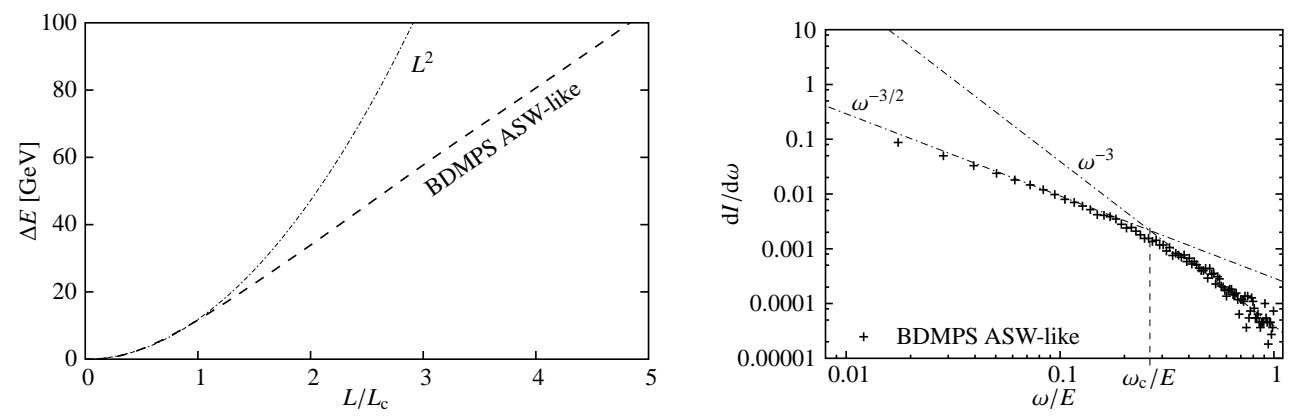

Figure 1: LHS: Total energy loss due to gluon radiation as a function of the path length $L$ in the medium. RHS: Gluon energy spectrum at $L=L_{\mathrm{c}} / 2$. 'BDMPS ASW-like' denotes a setup of the MC simulation that parallels as much as possible the assumptions of the analytical BDMPS ASW calculation. The parameter choice corresponds to $\hat{q} \approx 1 \mathrm{GeV}^{2} / \mathrm{fm}$, the projectile energy is $E=100 \mathrm{GeV}$.

The analytical calculations [2,3] are carried out in the kinematical regime $E \gg \omega \gg k_{\perp}, q_{\perp}>$ $\Lambda_{\mathrm{QCD}}$. In order to reproduce the results of these calculations these approximations have to be respected by the MC. As a consequence, the energy degradation of the projectile is neglected, the medium transfers only transverse momentum to the gluon, the momentum transfer is soft, the scattering centre takes no recoil and the gluon $k_{\perp}$ in the initial production process has to be small. We will discuss the consequences of these approximations in more detail in section 3 .

Characteristics for the BDMPS-ASW calculation $[2,3]$ are the modification of the gluon spectrum and the path length dependence of the energy loss. The gluon spectrum, which follows $\mathrm{d} I / \mathrm{d} \omega \propto \omega^{-1}$ for incoherent production, is modified to $\mathrm{d} I / \mathrm{d} \omega \propto \omega^{-3 / 2}$ for $\omega<\omega_{\mathrm{c}}$ and $\omega^{-3}$ for $\omega>\omega_{\mathrm{c}}$. The characteristic gluon energy $\omega_{\mathrm{c}} \simeq \hat{q} L^{2} / 4$ is the highest gluon energy that can be radiated coherently in a medium of length $L$. The energy loss $\Delta E$ increases quadratically with $L$ 
for $L<L_{\mathrm{c}}$, for $L>L_{\mathrm{c}}$ it becomes linear, $L_{\mathrm{c}} \simeq \sqrt{4 E / \hat{q}}$ is the formation time corresponding to the highest kinematically allowed gluon energy.

Figure 1 shows the results of our MC simulation for the energy loss and the gluon spectrum in the coherent regime at $L=L_{\mathrm{c}} / 2$. The results show the features expected from the analytical BDMPS-ASW calculation.

\section{Going Beyond the BDMPS-ASW Approximation}

The MC simulation is a tool that allows one to go beyond the approximations discussed in the previous section. We successively relax the assumptions to arrive at a more realistic description of radiative energy loss. The steps are

1. relax soft gluon approximation: the full power law tail of the elastic cross section is taken into account

$$
\frac{\mathrm{d} \sigma}{\mathrm{d} q_{\perp}^{2}} \propto \frac{1}{\left(q_{\perp}^{2}+\mu^{2}\right)^{2}} \theta\left(q_{\perp}^{2}-4 \mu^{2}\right) \quad \rightarrow \quad \frac{1}{\left(q_{\perp}^{2}+\mu^{2}\right)^{2}}
$$

2. energy conservation: the degradation of the projectile energy due to gluon radiation is accounted for

3. realistic kinematics of inelastic process: the gluon is produced with a transverse momentum $k_{\perp}$ distributed as $1 / k_{\perp}^{2}$ (as suggested, for instance, by full inelastic matrix elements); without the full kinematics introduced in the next step this may be seen as a rather extreme choice

4. recoil: the scattering centre in elastic and inelastic scattering events becomes dynamic and takes recoil; to arrive at a JEWEL-like scenario elastic scattering of the projectile also has to be included

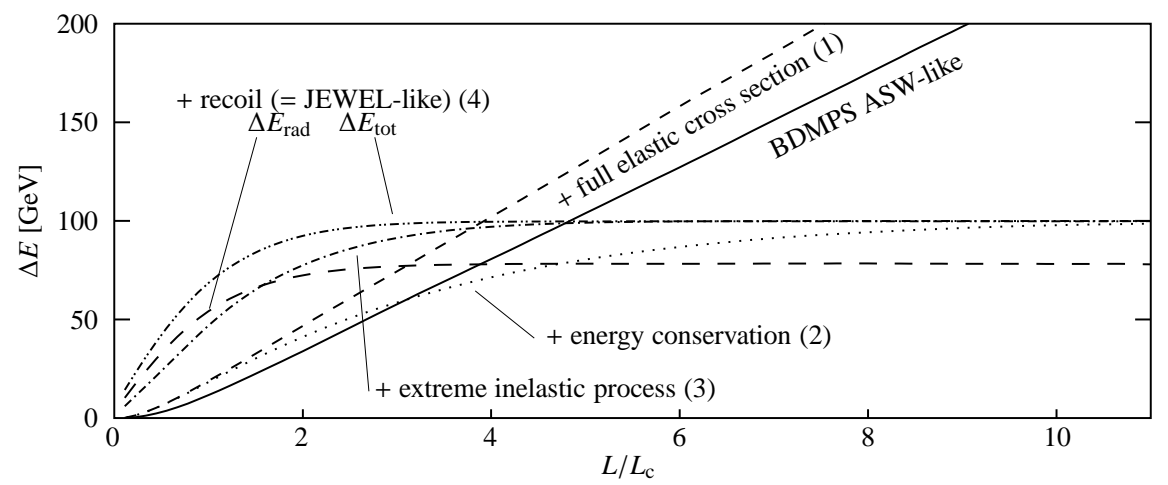

Figure 2: Energy loss as function of in-medium path length for the stepwise relaxation of assumptions. In the JEwEL scenario a distinction between radiative energy loss and total energy loss (including the energy taken by recoiling scattering centres) has to be made.

The parameter $\mu$ and the mean free path $\lambda$ are kept fixed, only in the Jewel scenario the elastic cross section is characterised by the infra-red regulator and the momentum distribution of the scattering centres. The energy loss and gluon spectrum for the different stages are shown 


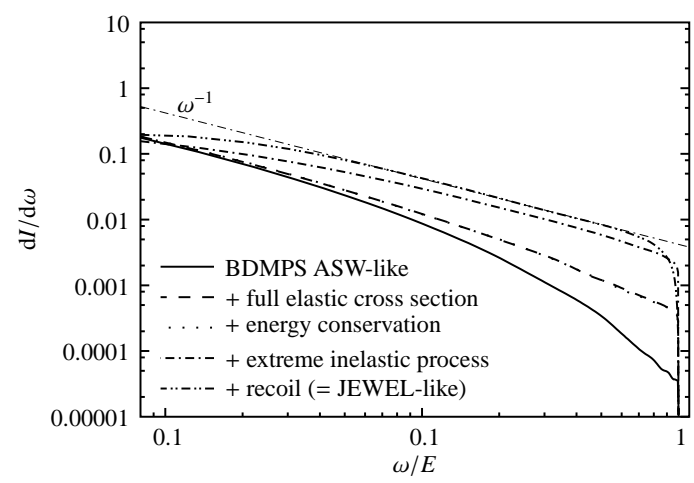

Figure 3: Gluon energy spectrum at $L=L_{\mathrm{c}} / 2$ for the stepwise relaxation of assumptions. Note that energy conservation plays only a minor role in this regime, so that the third lines lies on top of the second.

in figures 2 and 3. Energy conservation turns out to be an important effect $L>L_{\mathrm{c}}$, but plays a negligible role in the coherent regime for $L<L_{\mathrm{c}}$. Generally, the radiation looks more and more incoherent. The modifications of the elastic and inelastic cross sections lead to a loss of coherence. This is due to fluctuations that allow for much smaller formation times, i.e. large momentum transfers in elastic rescattering or large gluon $k_{\perp}$ in the inelastic process. The approximation $k_{\perp} \ll \omega$ in the BDMPS ASW calculation leads to long gluon formation times after the inelastic process and the soft scattering approximation inhibits a fast decoherence. In that sense the analytical calculation ensures maximal LPM-suppression, which can perhaps partly explain the large values of $\hat{q}$ that seem to be necessary to describe RHIC jet quenching data.

\section{Outlook}

We discussed how medium induced gluon radiation showing LPM interference can be implemented in a MC event generator without modifying the gluon emission process. This is an important step towards a dynamically consistent implementation of radiative energy loss. However, in order to arrive at a complete description of jet evolution in dense QCD matter, the algorithm has to be generalised to induced gluon emission in the presence of vacuum radiation. In this case there is interference between vacuum and medium induced radiation in addition to the interference between subsequent induced emissions. An appropriate MC algorithm generalising the prescription discussed here can be constructed and will be implemented in JEwEL. This will complete the description of interactions with the medium in JewEL.

\section{Acknowledgments}

This work was supported by the Alliance Program of the Helmholtz Association (HA216/EMMI).

\section{References}

[1] K. Zapp, G. Ingelman, J. Rathsman, J. Stachel and U. A. Wiedemann, Eur. Phys. J. C 60 (2009) 617

[2] U. A. Wiedemann, Nucl. Phys. B 588 (2000) 303.

[3] R. Baier, Y. L. Dokshitzer, A. H. Mueller, S. Peigné and D. Schiff, Nucl. Phys. B 484 (1997) 265.

[4] K. Zapp, J. Stachel and U. A. Wiedemann, arXiv:0812.3888 [hep-ph]. 